\title{
Statistical analysis of harmonic signals for testing of Electronic Devices
}

\author{
S.Herasimov $^{1}$,E.Roshchupkin ${ }^{2}$, V.Kutsenko ${ }^{3}$, S.Riazantsev $^{4}$, Yu.Nastishin ${ }^{5}$ \\ ${ }^{1}$ Ivan KozhedubKharkiv National Air Force University, Kharkiv, Ukraine, gsvnr@ukr.net \\ ${ }^{2}$ Ivan KozhedubKharkiv National Air Force University, Kharkiv, Ukraine, reszzz76@ gmail.com \\ ${ }^{3}$ Ivan KozhedubKharkiv National Air Force University, Kharkiv, Ukraine, xarley78@i.ua \\ ${ }^{4}$ Ivan KozhedubKharkiv National Air Force University, Kharkiv, Ukraine, sambor_2008@ukr.net \\ ${ }^{5}$ Hetman Petro Sahaidachnyi National Army Academy, Lviv, Ukraine, nastyshyn_yuriy@yahoo.com
}

\begin{abstract}
Harmonic signals are often used for control of functional state of electronic devices. In this paper we show that the goal can be achieved via monitoring of the statistical behavior of harmonic signals propagating through the tested device. Malfunctions of the device lead to the changes in the statistical characteristics of the harmonic signals and thus are used for the monitoring of the technical state of the device. We consider statistical behavior of such signals at the variation of their phase, caused by malfunctions in electronic devices. Deviation of the mathematical expectation from its nominal values and dispersion are proposed for estimation of malfunction of a device. A functional scheme of a controller analyzing the mathematical expectation and dispersion is proposed. An experiment demonstrating how the proposed approach can be used for monitoring of the technical state of an electronic device under the influence of the external noise is presented.
\end{abstract}

Key words : harmonic signals, malfunctions in electronic devices, maintenance testing, statistical characteristics of errors.

\section{INTRODUCTION}

Nowadays, when electronics deeply penetrates in all spheres of human being via electronic devices from smart pocket devices to sophisticated computerized and robotized networks of systems with elements of artificial intellect, in many cases control of their proper functionality becomes of survival importance. Despite vast variety of such devices, which differ essentially by their functions, operation and design, commonly all of them are based on typical elementary electronic modules, such as power supplies, signal generators, amplifiers, filters, triggers, modulators, etc., and thereby their functional state can be monitored with universal controllers. Development of universal testing techniques capable for monitoring of electronic schemes and design of corresponding controllers are in great demand.

\section{LITERATURE REVIEW}

Testing of electronic schemes with harmonic signals [1] - [4] is one of the possibilities for universalization of testing protocols. The principle of such an approach is as following. A harmonic signal from a generator is applied to the input of the inspected part of the electronic scheme of the device and the output signal is compared to the expected standard signal [5], [6]. In this paper the input testing signal is proposed to be of the form $\cos ^{k}(n \cdot \varphi+\delta \cdot \pi / 2)$, where $k$ and $n$ are natural (positive integer) numbers, such that $n$ denotes the multiplicity of the harmonic function, $\varphi$ is the phase increment, measured in angular units (radians or degrees) and $\delta$ takes the value 0 or 1 for the cosines or sinusoidal signals, respectively. Depending on the type of the inspected module, the output signal differs (usually by its phase and amplitude) from the input signal, but is expected to be of a form, which is standard for a given unit. In the case of malfunction the output signal is affected additionally. Analysis of the deviation of the output signal from the expected standard form is used for conclusion on the technical state of the inspected unit [6].

The statistical behavior of the parameter $\varphi$ of the harmonic function was studied in [7] - [11].

However, in most practical cases, the parameter $\varphi$ per se is of no importance. Instead, the value of its harmonic function is of interest. The latter is related to a specific functioning mode of many devices (such as selsyns, encoders, phase detectors, etc.), which deal with the harmonic function, rather than with $\varphi$. The same is for "indirect measurements", at which the conclusion on the technical state of a device is also formulated via analysis of the measured valued of the harmonic function. Derivations of analytical expressions describing statistical behavior of the probing signal are labor- and time-consuming; final derived expressions in most cases appear to be lengthy and inapplicable for analytical analysis. For this reason traditionally approximations with serial expansions are employed. Truncation to the first term of the serial expansion of the output signal, combined with assumption of high "signal-to-noise" ( $\mathrm{s} / \mathrm{n})$ ratio, often results in acceptable predicted s/n ratios [12] - [15]. However, consideration of next expansion terms is obviously needed for essentially 
non-linear signals [16]. Statistical analysis of testing harmonic signals in terms of varying parameters is, thus, in demand. To the best of our knowledge, handy expressions for statistical characteristics of the tested values of signals with harmonically varying parameters are not available in the literature. As stated above the main reason for this are difficulties related to the analytical derivations and simplifications of the derived expressions to handy forms. In this paper we present analytical expressions for statistical characteristics of harmonic functions of errors varying normally in accordance with Gaussian distribution and propose a scheme of a universal controller, which employs the derived expressions for detection of malfunctions in electronic devices.

\section{MAIN MATERIAL}

Suppose, the indirectly measured parameter is of the form:

$$
\varphi=\varphi_{0}+\Delta \varphi,(1)
$$

where $\varphi_{0}$ is the initial value of the parameter, $\Delta \varphi$ is the normally varying noise error with the following characteristics of the normal Gaussian distribution for the probability density of the error [17], [18]:

$$
\left\{\begin{array}{l}
f(\Delta \varphi)=\frac{1}{\sqrt{(2 \pi)} \sigma_{\varphi}} \times \exp \left\{-\frac{\Delta \varphi^{2}}{2 \sigma^{2} \varphi}\right\} \\
M[\Delta \varphi]=0 \\
D[\Delta \varphi]=M\left[(\Delta \varphi-M[\Delta \varphi])^{2}\right]=\sigma_{\varphi}^{2} .
\end{array}\right.
$$

with commonly accepted notations for density probability $f(\Delta \varphi)$, mathematical expectation $M[\Delta \varphi]$ and dispersion $D[\Delta \varphi]$. It is obvious, that for the parameter, varying according to equation (1) one has:

$$
\left\{\begin{array}{l}
f(\hat{\varphi})=\frac{1}{\sqrt{(2 \pi)} \sigma_{\varphi}} \times \exp \left\{-\frac{\left(\hat{\varphi}-\varphi_{0}\right)^{2}}{2 \sigma_{\varphi}^{2}}\right\} \\
M[\hat{\varphi}]=\varphi_{0} \\
D[\hat{\varphi}]=M\left[(\hat{\varphi}-M[\hat{\varphi}])^{2}\right]=\sigma_{\varphi}^{2}
\end{array}\right.
$$

Our next step is to find $f\left[F_{i}(\varphi)\right], M\left[F_{i}(\varphi)\right]$ and $D\left[F_{i}(\varphi)\right]$ with $i$ varying from 1 to 4 such that

$$
\left\{\begin{array}{l}
F_{1}\left(\varphi_{n}\right)=\sin (n \cdot \varphi) \\
F_{2}\left(\varphi_{n}\right)=\cos (n \cdot \varphi) \\
F_{3}\left(\varphi_{n}\right)=\sin ^{2}(n \cdot \varphi) \\
F_{4}\left(\varphi_{n}\right)=\cos ^{2}(n \cdot \varphi)
\end{array}\right.
$$

with $n$ being a certain natural number standing for the order (also called multiplicity) of the harmonic and $\varphi_{n}=n \cdot \varphi$ denoting the variable of the harmonic function of the $n$-th order.

The algorithm for derivation of the probability density of a function of an independent random variable (in its conventional sense defined in statistics) [8], [18]is of the form:

$$
f\left[F_{i}(\varphi)\right]=f\left[F_{i}^{-1}(\varphi)\right] \cdot\left|\frac{\partial\left[F_{i}^{-1}(\varphi)\right]}{\partial \varphi}\right|(5)
$$

where $F_{i}^{-1}(\varphi)$ is the function, which is inverse to $F_{i}(\varphi)$. Substitution of equation (4) in equation (5) gives the probability densities of harmonic functions $F_{i}\left(\varphi_{n}\right)$ for the variable parameter, equation (4):

$$
\left\{\begin{array}{l}
f\left(\sin \left(\varphi_{n}\right)\right)=\frac{1}{\sqrt{(2 \pi)} n \sigma_{\varphi} \sqrt{1-\sin ^{2}(n \cdot \varphi)}} \times \\
\times \exp \left\{-\frac{\left(\arcsin (\sin (n \cdot \varphi))-n \cdot \varphi_{0}\right)^{2}}{2 n^{2} \sigma^{2} \varphi}\right\} ; \\
f\left(\cos \left(\varphi_{n}\right)\right)=\frac{1}{\sqrt{(2 \pi)} n \sigma_{\varphi} \sqrt{\left[1-\cos ^{2}(n \cdot \varphi)\right]}} \times \\
\times \exp \left\{-\frac{\left(\arccos (\cos (n \cdot \varphi))-n \cdot \varphi_{0}\right)^{2}}{2 n^{2} \sigma^{2} \varphi}\right\} ; \\
f\left(\sin { }^{2}\left(\varphi_{n}\right)\right)=\frac{1}{\sqrt{(2 \pi)} n \sigma_{\varphi} \sqrt{\left[1-\sin ^{2}(n \cdot \varphi)\right] \cdot \sin ^{2}(n \cdot \varphi)}} \times \\
\times \exp \left\{-\frac{\left(\arcsin \left(\sqrt{\sin ^{2}(n \cdot \varphi)}\right)-n \cdot \varphi_{0}\right)^{2}}{2 n^{2} \sigma^{2} \varphi}\right\} ; \\
f\left(\cos { }^{2}\left(\varphi_{n}\right)\right)=\frac{1}{n \cdot \sqrt{(2 \pi)} n \sigma_{\varphi} \sqrt{\left[1-\cos ^{2}(n \cdot \varphi)\right] \cdot \cos ^{2}(n \cdot \varphi)}} \times \\
\times \exp \left\{-\frac{\left(\arccos \left(\sqrt{\cos ^{2}(n \cdot \varphi)}\right)-n \cdot \varphi_{0}\right)^{2}}{2 n^{2} \sigma^{2} \varphi}\right\} .
\end{array}\right.
$$

It should be noticed that the inverse trigonometric functions in equation (6) are multivalued, such that their main values are limited as following

$$
-\frac{\pi}{2} \leq \arcsin \left(\alpha_{k}\right) \leq+\frac{\pi}{2}, 0 \leq \arccos \left(\alpha_{k}\right) \leq \pi \text {. (7) }
$$

Mathematical expectation and dispersion as functions of $F_{i}\left(\varphi_{n}\right)$ are derived using the following expressions [18]

$$
\left\{\begin{array}{c}
M\left[\Phi_{i}\left(\hat{\varphi}_{n}\right)\right]=\int \Phi_{i}\left(\hat{\varphi}_{n}\right) \cdot f(\hat{\varphi}) \cdot d \hat{\varphi} ; \\
D\left[\Phi_{i}\left(\hat{\varphi}_{n}\right)\right]=\int\left[\Phi_{i}\left(\hat{\varphi}_{n}\right)\right]^{2} f(\hat{\varphi}) d \hat{\varphi}-\left(M\left[\Phi_{i}\left(\hat{\varphi}_{n}\right)\right]\right)^{2} .
\end{array}\right.
$$

Calculated mathematical expectations and dispersions are presented in Table 1 . 
S.Herasimov et al., International Journal of Emerging Trends in Engineering Research, 8(7), July 2020, 3791- 3798

Table 1: Statistical characteristics for harmonic functions

\begin{tabular}{|c|c|c|}
\hline$F_{i}\left(\varphi_{n}\right)$ & $M\left[F_{i}\left(\varphi_{n}\right)\right]$ & $D\left[F_{i}\left(\varphi_{n}\right)\right]$ \\
\hline $\sin (n \cdot \varphi)$ & $\sin \left(n \cdot \varphi_{0}\right) \cdot \exp \left\{-\frac{\left(n \cdot \sigma_{\varphi}\right)^{2}}{2}\right\}$ & $\begin{array}{l}M\left[\sin ^{2}(n \cdot \varphi)\right]-(M[\sin (n \cdot \varphi)])^{2}= \\
=\frac{1-\cos \left(2 \cdot n \cdot \varphi_{0}\right) \cdot \exp \left\{-2 \cdot\left(n \cdot \sigma_{\varphi}\right)^{2}\right\}}{2}- \\
-\sin ^{2}\left(n \cdot \varphi_{0}\right) \cdot \exp \left\{\left(n \cdot \sigma_{\varphi}\right)^{2}\right\}\end{array}$ \\
\hline $\cos (n \cdot \varphi)$ & $\cos \left(n \cdot \varphi_{0}\right) \cdot \exp \left\{-\frac{\left(n \cdot \sigma_{\varphi}\right)^{2}}{2}\right\}$ & $\begin{array}{l}M\left[\cos ^{2}(n \cdot \varphi)\right]-(M[\cos (n \cdot \varphi)])^{2}= \\
=\frac{1+\cos \left(2 \cdot n \cdot \varphi_{0}\right) \cdot \exp \left\{-2 \cdot\left(n \cdot \sigma_{\varphi}\right)^{2}\right\}}{2}- \\
-\cos ^{2}\left(n \cdot \varphi_{0}\right) \cdot \exp \left\{\left(n \cdot \sigma_{\varphi}\right)^{2}\right\}\end{array}$ \\
\hline $\sin ^{2}(n \cdot \varphi)$ & $\frac{1-\cos \left(2 \cdot n \cdot \varphi_{0}\right) \cdot \exp \left\{-2 \cdot\left(n \cdot \sigma_{\varphi}\right)^{2}\right\}}{2}$ & $\begin{array}{l}M\left[\sin ^{4}(n \cdot \varphi)\right]-\left(M\left[\sin ^{2}(n \cdot \varphi)\right]\right]^{2}= \\
=\frac{1-\cos ^{2}\left(2 \cdot n \cdot \varphi_{0}\right) \cdot \exp \left\{-\left(2 \cdot n \cdot \sigma_{\varphi}\right)^{2}\right\}}{4} \\
-\frac{1-\cos \left(4 \cdot n \cdot \varphi_{0}\right) \cdot \exp \left\{-2 \cdot\left(2 \cdot n \cdot \sigma_{\varphi}\right)^{2}\right\}}{8}\end{array}$ \\
\hline $\cos ^{2}(n \cdot \varphi)$ & $\frac{1+\cos \left(2 \cdot n \cdot \varphi_{0}\right) \cdot \exp \left\{-2 \cdot\left(n \cdot \sigma_{\varphi}\right)^{2}\right\}}{2}$ & $\begin{array}{l}M\left[\cos ^{4}(n \cdot \varphi)\right]-\left(M\left[\cos ^{2}(n \cdot \varphi)\right]\right)^{2}= \\
=\frac{1-\cos ^{2}\left(2 \cdot n \cdot \varphi_{0}\right) \cdot \exp \left\{-\left(2 \cdot n \cdot \sigma_{\varphi}\right)^{2}\right\}}{4} \\
-\frac{1-\cos \left(4 \cdot n \cdot \varphi_{0}\right) \cdot \exp \left\{-2 \cdot\left(2 \cdot n \cdot \sigma_{\varphi}\right)^{2}\right\}}{8}\end{array}$ \\
\hline
\end{tabular}

Derived expressions for systematic errors are of the form

$$
\left\{\begin{array}{l}
\Delta M\left[\sin \left(\varphi_{n}\right)\right]=\sin \left(\varphi_{0 n}\right)\left(1-\exp \left\{-\frac{\left(n \sigma_{\varphi}\right)^{2}}{2}\right\} ;\right. \\
\Delta M\left[\cos \left(\varphi_{n}\right)\right]=\cos \left(\varphi_{0 n}\right)\left(1-\exp \left\{-\frac{\left(n \sigma_{\varphi}\right)^{2}}{2}\right\} ;\right. \\
\Delta M\left[\sin ^{2}\left(\varphi_{n}\right)\right]=-\frac{\cos \left(2 \varphi_{0 n}\right)}{2}\left(1-\exp \left(-2\left(n \sigma_{\varphi}\right)^{2}\right) ;\right. \\
\Delta M\left[\cos ^{2}\left(\varphi_{n}\right)\right]=\frac{\cos \left(2 \varphi_{0 n}\right)}{2}\left(1-\exp \left(-2\left(n \sigma_{\varphi}\right)^{2}\right),\right.
\end{array}\right.
$$

\section{RESULTS AND DISCUSSIONS}

Analysis of statistical characteristics of the functions $F_{i}\left(\varphi_{n}\right)$, given in Table 1 and by equation (9), shows that the mathematical expectations are biased (displaced) with respect to the true values of the functions. The systematic error (displacement) $\Delta M\left[F_{i}\left(\varphi_{n}\right)\right]$ dependson the dispersion $\sigma_{\varphi}$ of the disturbing action (the normal error caused by the noise), on the true value of the harmonic function $F_{i}\left(n \cdot \varphi_{0}\right)$ as well as on the multiplicity of the testing signal $n$.Dependencies of systematic errors and dispersions for functions $\sin (n \cdot \varphi)$ and $\sin ^{2}(n \cdot \varphi)$ on the initial phase $\varphi_{0}$ for two values of $\sigma_{\varphi}$ at $n=1$ are plotted in Figure 1.

The statistical characteristics of errors for harmonic functions are of a well pronounced nonlinear oscillating character. The sum of squares of systematic errors of functions is given by:

$\Delta M^{2}\left[\sin \left(\varphi_{n}\right)\right]+\Delta M^{2}\left[\cos \left(\varphi_{n}\right)\right]=\left(1-\exp \left\{-\frac{\left(n \cdot \sigma_{\varphi}\right)^{2}}{2}\right\}\right)^{2}$

and the systematic errors of the values of the second power of the harmonic functions are equal by absolute value and opposite by their signs, i.e.

$$
\Delta M\left[\sin ^{2}\left(\varphi_{n}\right)\right]+\Delta M\left[\cos ^{2}\left(\varphi_{n}\right)\right]=0 .
$$

Dispersions of errors for the second power of harmonic functions $D\left[\sin ^{2}\left(\varphi_{n}\right)\right]$ and $D\left[\cos ^{2}\left(\varphi_{n}\right)\right]$ in contrast to the dispersions of errors for the first degree of harmonic functions $D\left[\sin \left(\varphi_{n}\right)\right]$ and $D\left[\cos \left(\varphi_{n}\right)\right]$ are equal for any value of $\varphi$.

Basing on the obtained results we propose a controller, the simplified scheme of which is shown in Figure 2. 
S.Herasimov et al., International Journal of Emerging Trends in Engineering Research, 8(7), July 2020, 3791- 3798

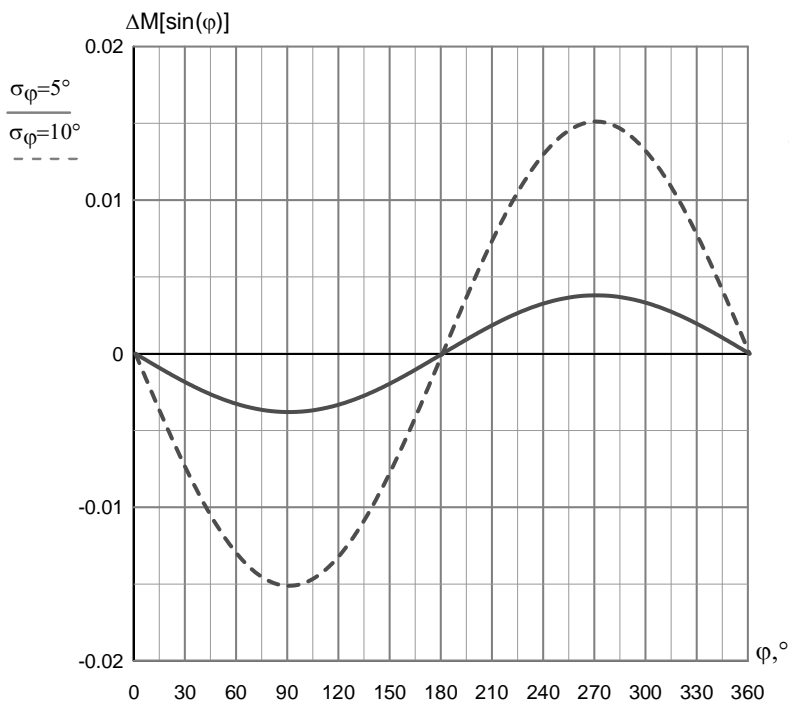

(a)

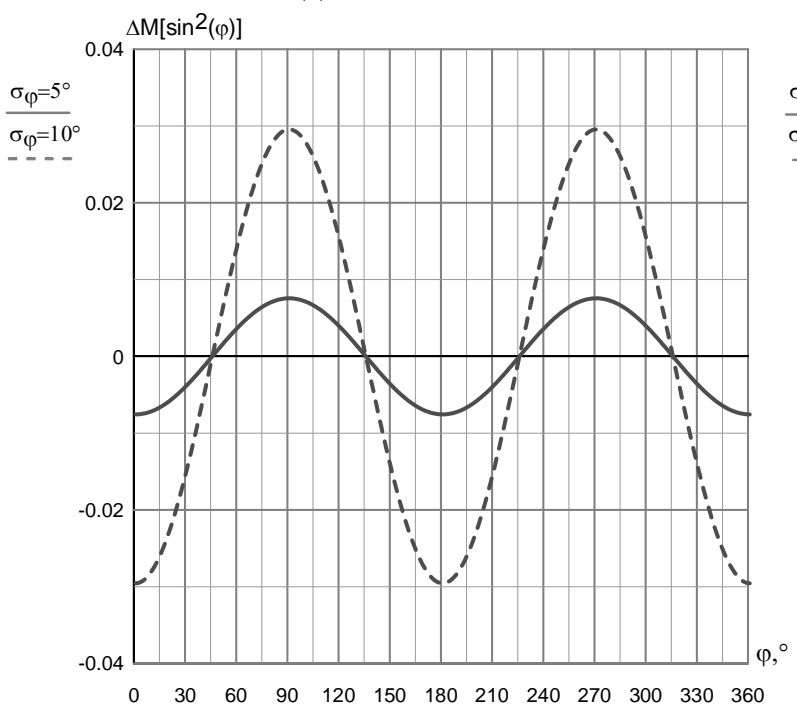

(c)

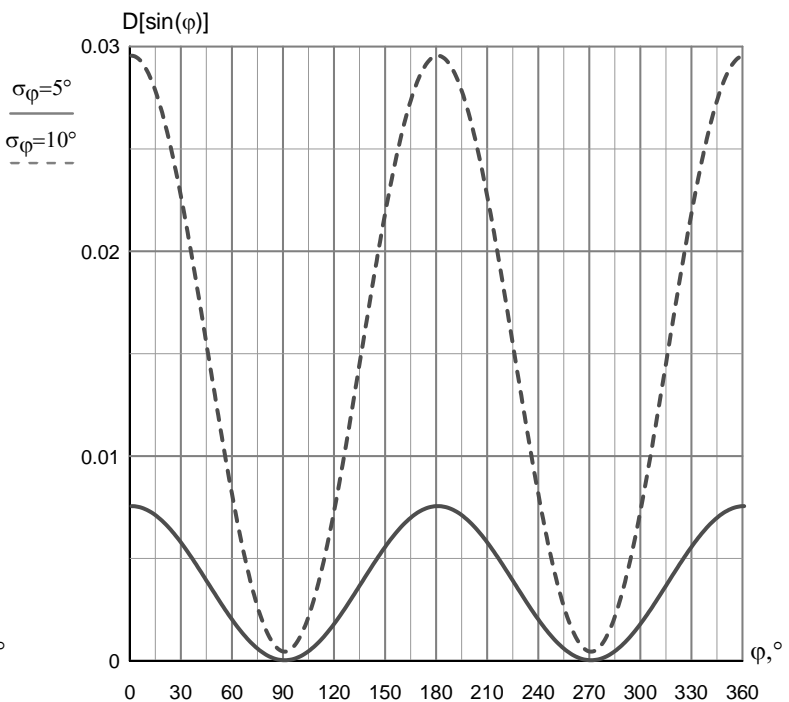

(b)

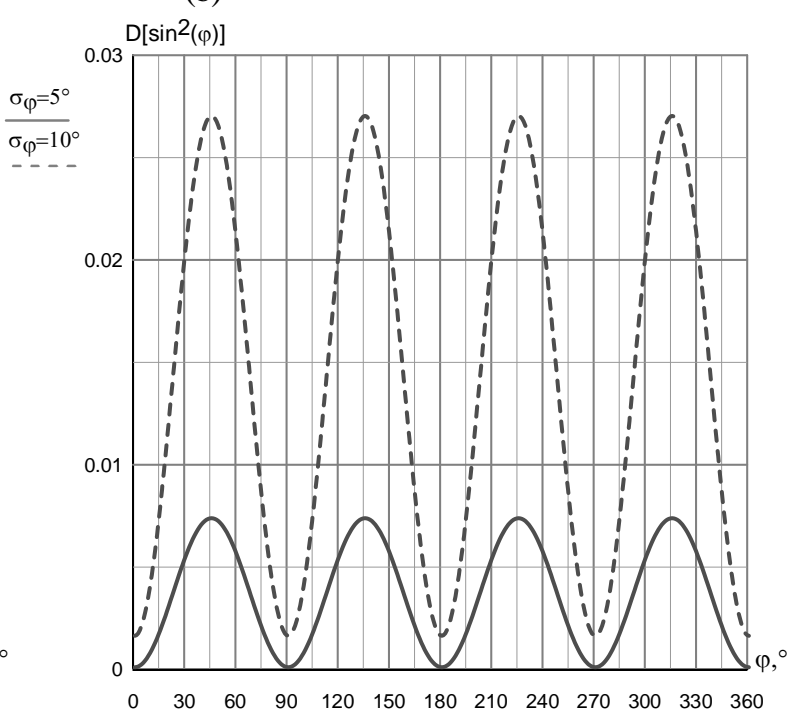

(d)

Figure 1: Dependencies of systematic errors $\Delta M$ (a, c) and dispersions $D(\mathrm{~b}, \mathrm{~d})$ for functions $\sin (n \cdot \varphi)$ and $\sin ^{2}(n \cdot \varphi)$, respectively, on the initial phase $\varphi_{0}$ for two values of $\sigma_{\varphi}=5$ (solid line) and $\sigma_{\varphi}=10$ (dashed line) at $n=1$

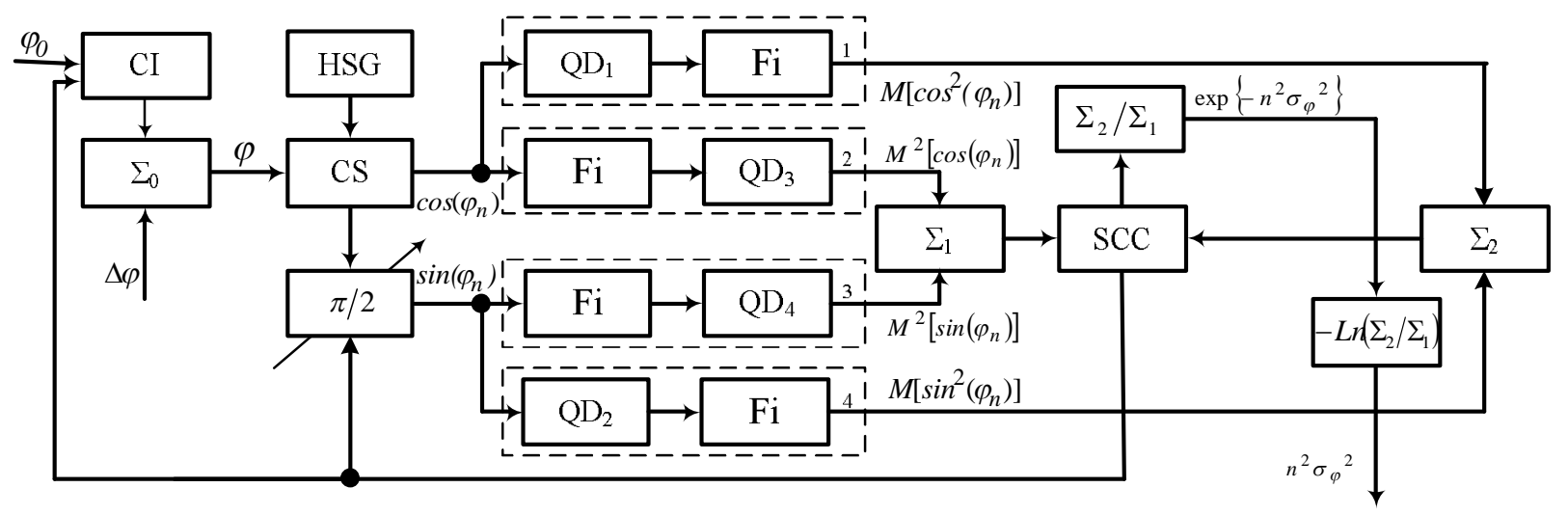

Figure 2: Simplified scheme of the controller 
The simplified scheme of the controller (Figure 2) consists of the following main components:

controlled inverter (CI); signal adder $\left(\Sigma_{i}\right)$; highly stable generator (HSG); converter of signals (CS); controlled $\pi / 2$ -phase-shift module; quadratic detector $\left(\mathrm{QD}_{i}\right)$; signal recorder (SR); signal voltage divider $\left(\Sigma_{2} / \Sigma_{1}\right)$; logarithmic amplifier ( - $\operatorname{Ln}\left(\Sigma_{2} / \Sigma_{1}\right)$; scheme of comparison and control (SCC).

Blocks 1-4, contoured in Figure 2 with a dashed line, include quadratic $\mathrm{QD}_{i}$ detectors, integrators $\mathrm{Fi}\left(\int_{0}^{T} F_{i}\left(\varphi_{n}\right)\right)$ and switching elements (not shown for simplicity). They differ from each other by the order of commutation of detectors and integrators, as well as by the order of commutation with the signal adders. It is not difficult to see that at the appropriate switching, blocks 1 and 2, 3 and 4 are equivalent, and the controller circuit, as such, can be reduced to a single channel with different modes of signal operation. The signal voltage divider $\left(\Sigma_{2} / \Sigma_{1}\right)$ and the logarithmic amplifier belong to the SCC, but for clarity of understanding of the described processes in Figure 2 they are depicted aside.

Let us consider the principle of operation of the proposed device (Figure 2). Suppose that the identity of the corresponding elements of the controller is set by default. In the absence of a useful signal (of indirectly measured parameter of the testing signal) by appropriate switching in the CCS, the noise level in channels of the controller is checked and necessary adjustment is performed. The testing signal $\varphi_{0}$, mixed with noise, enters the input of the signal converter (CS), the second input of which receives a signal from a highly stable generator (HSG). The signal, which is a harmonic function of the parameter $\varphi_{0}$ from the output of the converter, after branching, arrives to the quadrature channels, each of which also branches and arrives into channels for generation of the mathematical expectation of the square of the harmonic function and the square of the mathematical expectation of the harmonic function. The quadrature of the channels is achieved by introducing the $\pi / 2$-phase shift by the controlled phase-shift module.

Suppose that the signals are formed as shown in the diagram in Figure 2. Then, signals of the duration $T \cdot \exp \left\{-\left(n \cdot \sigma_{\varphi}\right)^{2}\right\}$ and $T$ from the output of the adders $\Sigma_{1}$ and $\Sigma_{2}$, respectively, are sent to the SCC, where $T$ is the known accumulation time. From the output of the divider $\Sigma_{2} / \Sigma_{1}$, a signal equal to $\exp \left\{-\left(n \cdot \sigma_{\varphi}\right)^{2}\right\}$ arrives to the logarithmic amplifier $-\operatorname{Ln}\left(\Sigma_{2} / \Sigma_{1}\right)$, from the output of which the voltage $\left(n \cdot \sigma_{\varphi}\right)^{2}$ is obtained. The value of the voltage at the output of the adder $\Sigma_{1}$, which appears to be different from $T$, indicates the non-quadrature character of the channels, i.e. the latter indicates the introduction of a $(\pi / 2+\xi)$-phase shift by the phase-shift module, where $\xi$ is the phase error. In such a case, the input of the controlled inverter receives a signal indicating about the change in the sign of the input useful signal. The difference between the direct and inverted signals from the outputs of the adders $\Sigma_{1}$ and $\Sigma_{2}$ in the SCC will be of the form

$$
\begin{gathered}
\Sigma_{1}: \Delta_{\Sigma_{1}}^{ \pm}=\left(M^{2}\left[\cos \left(\varphi_{n}\right)\right]+M^{2}\left[\sin \left(\xi+\varphi_{n}\right)\right]\right)- \\
\left(M^{2}\left[\cos \left(-\varphi_{n}\right)\right]+M^{2}\left[\sin \left(\xi-\varphi_{n}\right)\right]\right)= \\
=T \cdot \frac{4 \cdot \cos \left(\varphi_{0 n}\right) \cdot \sin \left(\varphi_{0 n}\right) \cdot \cos (\xi) \cdot \sin (\xi)}{\exp \left\{\left(n \cdot \sigma_{\varphi}\right)^{2}\right\}} ; \\
\Sigma_{2}: \Delta^{ \pm} \Sigma_{2}=M\left[\left(\cos ^{2}\left(\varphi_{n}\right)+\sin ^{2}\left(\xi+\varphi_{n}\right)\right)\right]- \\
M\left[\cos ^{2}\left(-\varphi_{n}\right)+\left(\sin ^{2}\left(\xi-\varphi_{n}\right)\right)\right]= \\
=T \cdot \frac{4 \cdot \cos \left(\varphi_{0 n}\right) \cdot \sin \left(\varphi_{0 n}\right) \cdot \cos (\xi) \cdot \sin (\xi)}{\exp \left\{2 \cdot\left(n \cdot \sigma_{\varphi}\right)^{2}\right\}}
\end{gathered}
$$

where the values of the functions $\cos \left(n \cdot \varphi_{0}\right)$ can be preliminarily estimated with the accuracy to a factor $\exp \left\{-\left(n \cdot \sigma_{\varphi}\right)^{2} / 2\right\}$ by the values of the voltages, taken from the outputs of the integrators of blocks 2-3, respectively. The logarithm of the ratio of the differences between the direct and inverted signals from the outputs of the adders $\Sigma_{1}$ and $\Sigma_{2}$ at the output of the logarithmic amplifier is given by

$$
-\operatorname{Ln}\left(\frac{\Delta^{ \pm} \Sigma_{2}}{\Delta^{ \pm} \Sigma_{1}}\right)=\left(n \cdot \sigma_{\varphi}\right)^{2}
$$

and does not depend on the phase error of the phase-shift module, which allows for the estimation of the dispersion of the error.

Thus, using the difference between the direct and inverted signals as a mismatch signal, the phase shift in the phase-shift module is eliminated, since $\left.\Delta^{ \pm} \rightarrow 0\right|_{\xi \rightarrow 0}$. The proposed scheme allows for control of the quadrature of the channels of the device, for measuring of the noise level as well as for the estimation of the dispersion of measurement errors, and can be used in existing and future control and monitoring systems. One of the particular advantages of the controller is that it maintains the phase shift between the input and output signals to be constant in time. With the proposed controller the corresponding testing measurements and calculations can be computerized.

The proposed theoretical approach is the theoretical tool for the estimation of the error values for signals with harmonically varying parameters and the designed scheme of the controller based on this approach allows one to maintain high accuracy of the phase characteristics of the phase detector in precision measuring instruments as well as to monitor the deviations in the technical state of the system under control by detecting changes in its phase characteristics. In electrical precision measuring devices, as well as in reactive electrical energy meters, it is important to maintain sufficient accuracy of the stabilization of equality of the characteristics of the input and output voltages under 
destabilizing factors and variation of parameters of the internal constructive elements. For example, at measurements of the power of the electrical energy it is quite important to ensure a phase shift of 90 degrees between the input and output voltages to reduce (eliminate) the methodical error.

One of the most significant disadvantages of the available technical solutions is that they do not guaranty the absolute identity of the phase characteristics of the input and output paths in such devices. The problem is rooted in different levels of internal noise of single paths; these differences appear due to the imperfect identity of the elemental base of different paths, and, thus, become the origin of the occurrence of methodical error.

Derived relationships, summarized in Table 1, show that the presence of errors in the output signal values with harmonically varying parameters is due to either the internal noise in the measuring channels or due to the variation in the phase shift in different paths. Thus, despite the mismatch in the elemental base of the different channels of the device, which lead to the occurrence of internal noise, by changing the phase shift between the input and output signals the proposed device (Figure2) diminishes the errors at the output, thereby maintaining high precision of the equality of the phase characteristics of the input and output signals (the voltage, in our case).

To illustrate the suitability of the derived expressions for control of the technical state of electronic devices we have performed an experiment using the set-up, the functional scheme of which is shown below its photograph in Figure 3. The elements of the scheme are indicated by the corresponding abbreviations in the scheme and on the photograph.
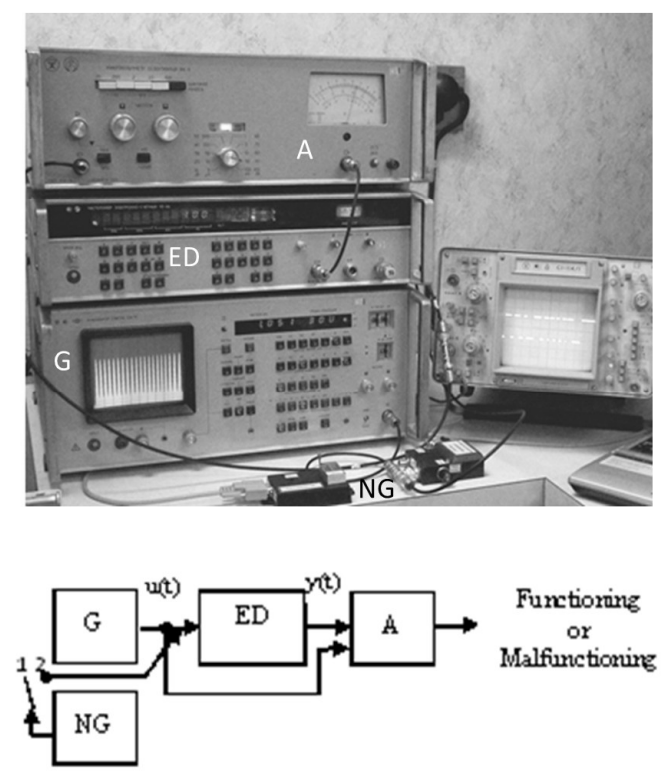

Figure 3:Experimental set-up: generator $(G)$, electronic device (ED), analyzer (A), noise generator (NG)

First, let the switch be in its position 1 (Figure 3). A harmonic signal of the voltage $u(t)$ and of the frequency $1 \mathrm{kHz}$
(Figure 4 a) is sent from the output of the generator, $G$ (Low-frequency precision generator of signals G3-110 made by Zavod Izmeritel, St. Petersburg, Russia), to the inputs of the tested electronic device, ED (here the amplifier of signals, made by Etalon-Prylad, Kharkiv, Ukraine) and to the signal analyzer, A (Spectrum analyzer C4-74, made by OJSC "Meridian" named after S. P. Korolev, Kyiv, Ukraine,). The signal $y_{1}(t)$ from the output of the ED is sent to the second input of the analyzer (Figure 3). The oscillograms of the signals (Figure 4a,b) are recorded using the oscilloscope (Universal Oscilloscope, C1-114/1, made by "Zavod Belit", Minsk, Belorussia).

Since the same generator $(\mathrm{G})$ is used to form the signals $u(t)$ (Figure4a,b) and $y_{1}(t)$ (Figure4b), which are sent to the inputs of the tested electronic device and analyzer, respectively, we assume that these two signals are identical by their shape, though possibly being of different amplitudes, and that the phase shift between them is constant in time (Figure $4 \mathrm{~b}$ ). Then, inside the analyzer, the difference

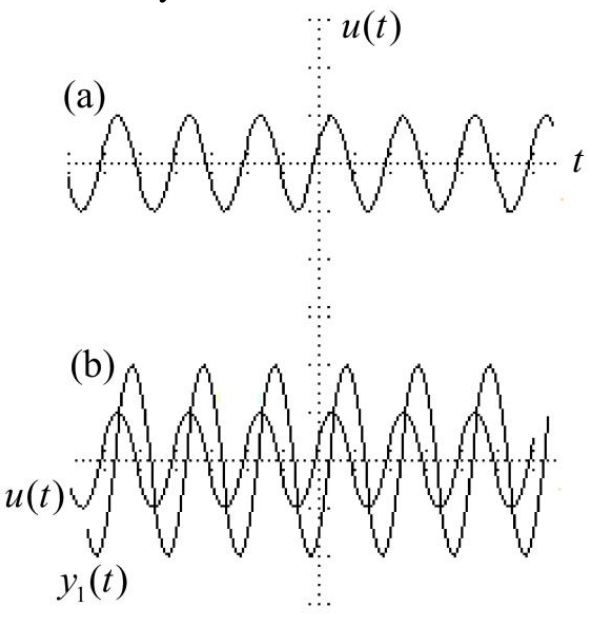

Figure 4: Shape of the signals $u(t)$ and $y_{1}(t)$

between the signals $u(t)$ and $y_{1}(t)$ is expectedto be affected by the internal noise of the electronic device; in other words this difference reflects the technical state of the device (in this case, either properly functioning or malfunctioning). Here we choose, the phase difference to be $90^{\circ}$ (Figure $4 \mathrm{~b}$ ). Using the derived relations (Table 1), we calculate the possible errors of the values of signals with harmonically varying parameters after passing through the electronic device: $\Delta M[\sin (\varphi)]=0,003^{\circ}, D[\sin (\varphi)]=0,0$, which indicate that the internal noise inside the tested device is negligibly small. Then, the switch is set into its position 2, connecting the noise generator (Test pattern generator G6-36 made in Russia) and, threby, modelling the signal, given by equationError! Reference source not found.. In other words by connecting the noise generator we add a disturbing effect. Thus, the input signal becomes $u_{2}(t)$. As a result, in the analyzer we obtaine another difference between the input signal $u(t)$ and the output signal $y_{2}(t)$, because the signal 
$y_{2}(t)$ contains an introduced disturbance (Figure 5). Series of measurements of the phase difference between the signals $u(t)$ and $y_{2}(t)$ using an analyzer C4-74 (Figure $5 \mathrm{c}$ ) give the average value $\Delta \varphi=12,1^{\circ}$. The systematic error (offset) $\Delta M[\sin (\varphi)]=1,12^{\circ}$ depends on the disdturbing action (noise), equationError! Reference source not found.. The difference in the error values of signals with harmonically varying parameters after passing through an electronic device before and after the application of disturbane (noise) shows that the characteristics of the electronic device have changed. Thus, the results of the experiment confirm the capability of the proposed method to control the technical state of electronic devices.

In addition, the circuit (Figure 3) allows one to measure the effects of external noise disturbance on the functioning of electronic devices.

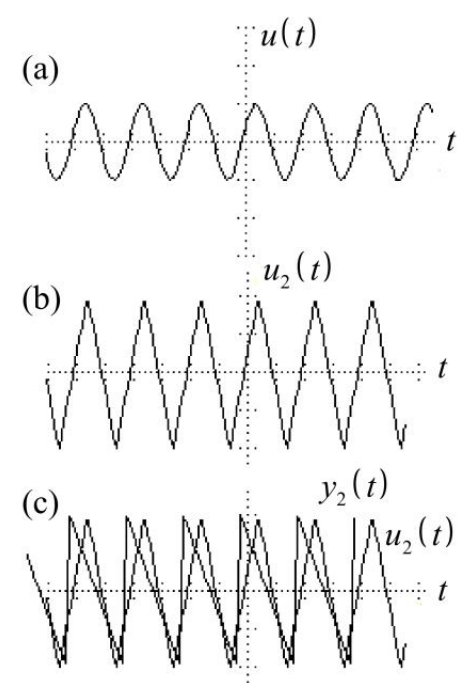

Figure 5: Shape of the signals $u(t), u_{2}(t)$ and $y_{2}(t)$

It remains to discuss the advantages of the testing signals of the form $\cos ^{k}(n \cdot \varphi+\delta \cdot \pi / 2)$ with $k>1$ in comparison with traditionally used periodical signals with $k=1$. An example of application of the testing signals with $k=2$ is related to the functional specifics of some measuring devices such as power and energy meters, where by definition the testing signal is squared on its propagation through the measuring device.

Concerning the values $k>2$, we refer to our recent paper [6], where the general criteria of optimality for the synthesis of testing signals for electronics have been suggested. According to these criteria, a testing signal should provide maximums for the indices of information and sensitivity together with the minimum of the mean square error. We have shown that in the presence of Gaussian electromagnetic noise these three criteria reduce into a single criterion, given by in [6]. Explicitly this criterion corresponds to the maximum of a function, which is proportional to the matrix $B_{i j}$ composed of derivatives of the testing signal over the sensitive variables (see [6] for details). For a general case of the testing signal of the form $\cos ^{k}(n \cdot \varphi+\delta \cdot \pi / 2)$, the exact form of the matrix $B_{i j}$ is not available yet. The corresponding derivations are labor- and time-demanding and will be a subject for consideration in our future paper. Nevertheless, some general properties of the $B_{i j}$ matrix are obvious. Indeed, since by the definition, the components of $B_{i j}$ are functions of derivatives of the testing signal, they will be proportional to the power $k$ of the testing signal. Therefore, higher values of $k$ imply higher sensitivity of the testing signal. Analytical expressions for statistical characteristics of the harmonic functions for $k=1,2$ are presented in this paper. Absence of the corresponding handy expressions for $k>2$ encourages future studies in this direction.

It should be also noted that the employment of the form $\cos ^{k}(n \cdot \varphi+\delta \cdot \pi / 2)$ for the testing signal can be a requirement for the inspection of electronic systems of devices connected into a network. In such a case higher orders of $k$ imply higher values for the so-called index of information [6] and will be required to find the correlation relations between the individual parameters of the single components of the system on the one hand and the control parameters of the electronic system as a whole on the other.

\section{CONCLUSIONS}

We have derived expressions for mathematical expectation $M$, dispersion $D$ and the error $\Delta M$ for four harmonic functions $F_{i}\left(\varphi_{n}\right)$ with the parameter $\varphi_{n}$ varying normally in accordance with the Gaussian distribution. On the basis of the obtained relationships, the scheme of the controller is proposed, which allows for testing of the channels quadrature, measuring the noise level and estimating the dispersion of the measurement errors. Obtained results can be used for testing of vast variety of existing and future systems of data processing, measuring and control systems.

The principles of many precision measuring devices and techniques, used for faults detections and their isolation, or more generally for the determination of the technical state of electronic devices, including the approach, described in this paper, require sufficient accuracy of the stabilization of equality of the characteristics of the input and output voltages under destabilizing factors and variation of parameters of the internal constructive elements. For example, it is quite important to ensure, that the phase shift between the input and output voltages, is constant in time. Such a function is provided by the proposed controller.

To illustrate the feasibility of the proposed approach for control of the technical state of electronic devices we have performed an experiment, the idea of which is to demonstrate how the proposed approach can be used to determine the error values for harmonically varying signals propagating through a real electronic device under a noise disturbance. Comparison 
of the calculated statistical parameters of the testing signal before and after the application of the noise reveals that these parameters are suitable and sensitive for the monitoring of the technical state of an electronic device. Using the derived relations summarized in Table 1 for the data measured in the experiment we have calculated the errors and corresponding dispersion of the values of signals with harmonically varying parameters after passing through the tested electronic device and found that before the application of the external noise, the internal noise inside the tested device is negligibly small. Acquisition and calculations of the corresponding data after application of the Gaussian noise revealed that the error $\Delta M$ and dispersion $D$ are indeed sensitive enough for the determination of the technical state of the tested electronic device. It is worth noting that this experiment, which we have done manually, can be computerized using the proposed controller.

To highlight the advantages of the form $\cos ^{k}(n \cdot \varphi+\delta \cdot \pi / 2)$ with $k>2$ we argue that higher values of $k$ correspond to the optimal criteria for testing signals and imply higher indices of sensitivity and information. The employment of the harmonic testing signal with $k=2$ is the consequence of the functional feature for some devices for which the signal is squared on its propagation through the device. Higher values of $k$ can be a mandatory requirement for complex electronic systems composed of devices connected into a network.

\section{REFERENCES}

1. H.Hajimolahoseini, M.R.Taban, and H.Soltanian-Zadeh. Extended Kalman Filter frequency tracker for nonstationary harmonic signals, Measurement, vol.45, p.p.126-132,2012 https://doi.org/10.1016/j.measurement.2011.09.008.

2. Dr. Sasi Bhanu J, Dr. Baswaraj D, Sunitha Devi Bigul, and Dr. JKR Sastry. Generating Test cases for Testing Embedded Systems using Combinatorial Techniques and Neural Networks based Learning Model, International Journal of Emerging Trends in Engineering Research, vol. 7, is. 7, p.p. 417-429, 2019 https://doi.org/10.30534/ijeter/2019/047112019.

3. F.Bonavolontà, M.D'Apuzzo, A.Liccardo, and G.Mieleb, Harmonic and interharmonic measurements through a compressed sampling approach, Measurement, vol.77, p.p. 1-15, 2016 https://doi.org/10.1016/j.measurement.2015.08.022.

4. Shin Kihong.On the Selection of Sensor Locations for the Fictitious FRF based Fault Detection Method, International Journal of Emerging Trends in Engineering Research, vol. 7, is. 7, p.p. 569-575, 2019, https://doi.org/10.30534/ijeter/2019/277112019.

5. V.M.Chinkov, and S.V.Gerasimov.Study and justification of the criteria of optimization of measurement signals for automated control systems, Ukrainian metrological journal, vol. 4, p.p. 43-47, 2013 http://www.metrology.kharkov.ua/fileadmin/user_uploa
d/data_gc/umj/2013/umj_2013_4/JRN/PDF/2.pdf.

6. S. Herasimov, V. Pavlii, O. Tymoshchuk and other.Testing Signals for Electronics: Criteria for Synthesis, Journal of Electronic Testing, vol. 35, is.148, p.p. $1-9,2019$

https://doi.org/10.1007/s10836-019-05798-9.

7. P.I.Dudnik, A.R.Ilchuk, and B. G. Tatarskii. Multifunctional radar systems: textbook for universities, Ed. B.G. Tatarskii, Moscow, Drofa, 2007.

8. Spatial-temporal signal processing, Ed. I. Kremer, Moscow, Radio and Communication, 1984.

9. N.Levanon, and E.Mozeson, Radar signals, Hoboken NJ, John Wiley \& Sons Inc., 411 p.,2004.

10. E.Murphy, andS.Colm.All About Direct Digital Synthesis, Analog Dialogue, vol. 8, is. 3, p.p.1-5, 2004,http://www.analog.com/en/analog-dialogue/article s/all-about-direct-digital-synthesis.html.

11. X.Wu, Z.Tian, T.Davidson, and G.Giannakis, "Optimal waveform design for UWB radios",IEEE Transactions on Signal Processing,vol.54, is. 6, p.p. 2009-2021, 2006,http://www.ece.mcmaster.ca/ davidson/pubs/Wu_ etal_UWB_waveform_design.pdf.

12. Yu.Rybin.Measuring Signal Generators, Theory and Design, Dordrecht, Heidelberg, London, New York, Springer, 2014.

13. C.W.Helstrom.Elements of Signal Detection and Estimation, Prentice Hall, Englewood Cliffs, NJ, USA, 1995.

14. S.Herasimov, Y. Belevshchuk, I. Ryapolovand other. Characteristics of radiolocation scattering of the SU-25T attack aircraft model at different wavelength ranges,Information and controlling systems. Eastern-European Journal of Enterprise Technologies, 6/9 (96), p.p. 22-29, 2018,https://doi.org/10.15587/1729-4061.2018.152740.

15. Sam Karimian-Azari, J. R. Jensen, andM. G. Christensen. Computationally efficient and noise robust DOA and pitch estimation, IEEE/ACM Transactions on Audioand Language Processing,vol.24,p.p. 1613-1625, 2016, https://doi.org/10.1109/TASLP.2016.2577501.

16. S. Herasimov, O. Tymochko, O. Kolomiitsev and other.Formation Analysis of Multi-Frequency Signals Of Laser Information Measuring System,EUREKA: Physics and Engineering, vol. 5, p.p.19-28, 2019 , https://doi.org/10.21303/2461-4262.2019.00984.

17. E.S.Ventzel.Theory of Probability: Textbook for high schools, Moscow, Vysshaya Shkola, 2001.

18. G. A.Korn, and T. M.Korn, Mathematical Handbook for Scientists and Engineers. New York. Dver Publications Inc., 2000. 